\title{
CONSENTIR À DES EXPÉRIENCES SEXUELLES SANS EN AVOIR ENVIE
}

La logique de redevabilité : responsabilité individuelle ou injonction sociale genrée ?

\author{
Myrian Carbajal, Annamaria Colombo et Marc Tadorian
}

Association Française des Anthropologues | «Journal des anthropologues »

2019/1 n 156-157 | pages 197 à 218

ISSN 1156-0428

Article disponible en ligne à l'adresse :

https://www.cairn.info/revue-journal-des-anthropologues-2019-1-page-197.htm

Distribution électronique Cairn.info pour Association Française des Anthropologues.

(C) Association Française des Anthropologues. Tous droits réservés pour tous pays.

La reproduction ou représentation de cet article, notamment par photocopie, n'est autorisée que dans les limites des conditions générales d'utilisation du site ou, le cas échéant, des conditions générales de la licence souscrite par votre établissement. Toute autre reproduction ou représentation, en tout ou partie, sous quelque forme et de quelque manière que ce soit, est interdite sauf accord préalable et écrit de l'éditeur, en dehors des cas prévus par la législation en vigueur en France. Il est précisé que son stockage dans une base de données est également interdit. 


\section{Journal des anthropologues}

Journal des

Association française des anthropologues

$156-157 \mid 2019$

Dettes de sexe?

\section{Consentir à des expériences sexuelles sans en avoir envie}

La logique de redevabilité : responsabilité individuelle ou injonction sociale genrée?

Consenting to Unwanted Sexual Experiences. The Logic of Indebtedness:

Individual Responsibility or Gendered Social Expectations?

\section{Myrian Carbajal, Annamaria Colombo et Marc Tadorian}

\section{OpenEdition Journals}

Édition électronique

URL : http://journals.openedition.org/jda/8244

DOI : $10.4000 /$ jda. 8244

ISSN : 2114-2203

\section{Éditeur}

Association française des anthropologues

\section{Édition imprimée}

Date de publication : 15 mai 2019

Pagination : 197-218

ISSN : 1156-0428

Distribution électronique Cairn

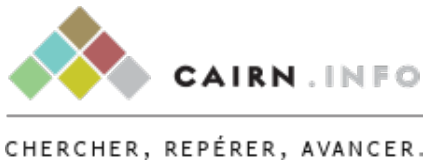

\section{Référence électronique}

Myrian Carbajal, Annamaria Colombo et Marc Tadorian, «Consentir à des expériences sexuelles sans en avoir envie », Journal des anthropologues [En ligne], 156-157 | 2019, mis en ligne le 01 janvier 2022, consulté le 22 octobre 2019. URL : http://journals.openedition.org/jda/8244 ; DOI : 10.4000/jda.8244 


\title{
CONSENTIR À DES EXPÉRIENCES SEXUELLES SANS EN AVOIR ENVIE La logique de redevabilité : responsabilité individuelle ou injonction sociale genrée ?
}

\author{
Myrian CARBAJAL* - Annamaria COLOMBO** \\ Marc TADORIAN***
}

\begin{abstract}
Ça m'est arrivé plusieurs fois d'avoir des rapports [sexuels] qui n'étaient pas consentis à $100 \%$. Et puis justement, $[\ldots]$ où je me sentais un petit peu, on va dire, obligée de faire ça à la personne, parce que je savais que c'était tout ce qu'elle attendait et puis qu'elle essaie de me faire absolument plaisir avec plein de choses et puis qu'à la fin je me suis dit, bon ben, ok. [...] Je me suis sentie redevable envers... (Joan ${ }^{1}, 17$ ans).
\end{abstract}

Dans le cadre d'une recherche portant sur les transactions sexuelles impliquant des jeunes en Suisse ${ }^{2}$, plusieurs jeunes femmes, comme Joan, ont exprimé avoir parfois consenti sans forcément en

* - ** - *** HES-SO, Haute école de travail social Fribourg

Courriel : myrian.carbajal@hefr.ch - annamaria.colombo@hefr.ch marc.tadorian@unine.ch

1 Tous les prénoms sont fictifs.

2 Recherche « Sexe, relations... et toi ?» menée à la HES-SO, Haute école de travail social Fribourg (HETS-FR) par A. Colombo et M. Carbajal, avec la collaboration de M. Carvalhosa Barbosa, C. Jacot, M. Tadorian et J.-L. Heeb et financée par la Fondation Oak. Plus d'informations sur www.sexe-et-toi.ch ou www.hets-fr.ch. 
avoir envie, mais par sentiment de redevabilité ${ }^{3}$, à s'engager dans des expériences sexuelles avec des hommes. Il s'agit d'expériences où des hommes, de manière ponctuelle, leur ont payé un verre, un repas ou encore offert l'hébergement à la suite d'une sortie en discothèque ou, de manière plus régulière, leur ont offert logement, nourriture ou autre. À travers l'analyse de cette logique de redevabilité, comprise comme révélatrice d'attentes sociales genrées, nous montrons dans cet article que le consentement ne peut pas être uniquement interprété en termes de responsabilité individuelle, mais qu'il s'inscrit dans une configuration sociale où la sexualité féminine est posée comme une dette ${ }^{4}$.

Après avoir situé la recherche de laquelle sont issues les données de cet article, nous mettons en lumière dans la deuxième section que ce qui peut apparaître comme un consentement à s'engager dans une relation intime relève davantage de « compromis » négociés entre les acteurs et actrices, au sens de la sociologie des transactions (Rémy, 1996 ; Schurmans, 2013). Nous définissons le compromis comme le résultat, temporaire et susceptible d'être remis en question, d'un ajustement entre liberté et contrainte. Dans la troisième section, nous soutenons que ces compromis prennent place dans des processus sociaux qui sont reliés, notamment, à un ordre « hétérosexuel» (Butler, 2005) qui détermine les attentes sociales en fonction du genre. Dans cet ordre normatif, la sexualité des femmes est posée comme une «dette de sexe », dans le sens qu'elle est considérée comme « due » en réponse aux besoins sexuels des hommes. Ce cadre normatif n'exclut pas les marges de manœuvre et la quatrième section met en lumière les stratégies de négociation développées par les jeunes. Enfin, nous

3 Ce sentiment de redevabilité a également été exprimé par quelques jeunes hommes dans le cadre de relations homosexuelles. Dans cet article, nous faisons le choix de nous concentrer sur les relations hétérosexuelles, où cette logique ressort de façon plus marquée.

4 Les auteures remercient les évaluateurs-trices de l'article pour leurs précieux commentaires qui ont contribué à approfondir ce concept de « dettes de sexe ». 
montrons dans la conclusion les limites d'une approche du consentement essentiellement axée sur une injonction à changer les comportements individuels, en particulier ceux des femmes, sans agir sur l'ordre hétérosexuel au sein duquel prennent place ces comportements.

\section{Comprendre le sens donné par les jeunes à leurs expériences de transactions sexuelles}

Cet article se base sur les résultats d'une recherche portant sur les transactions sexuelles, définies comme toutes expériences sexuelles associées à des échanges financiers, matériels et/ou symboliques (Broqua \& Deschamps, 2014 : 12). Cette étude privilégie une approche compréhensive de la sexualité (Gagnon \& Simon, 1973), qui valorise les logiques subjectives qui sous-tendent les interactions liées à la sexualité. Ainsi, nous adoptons le postulat proposé par la psychologie sociale des représentations (Jodelet, 1989), selon lequel notre relation au monde et aux autres est structurée par les représentations sociales qui orientent et organisent les conduites sociales. Dans une démarche exploratoire, cette recherche visait à faire émerger les sens donnés à ces pratiques par les jeunes plutôt que de donner a priori une définition normative des transactions sexuelles, tout en s'appuyant sur un cadre théorique articulant des concepts issus de la sociologie de la sexualité (Bozon, 2002; Combessie \& Mayer, 2013) et de la sociologie de la transaction sociale (Rémy, 1996 ; Schurmans, 2013).

Dans la perspective de la sociologie de la transaction sociale, les échanges sexuels peuvent être compris comme des formes de transaction qui impliquent «l'ajustement mutuel des actes de différents individus à l'intérieur du processus social» (Schurmans, 2013 : 88 ) ; processus dans lequel des formes variées de négociation sont à l'œuvre. Celles-ci se déroulent dans «des espaces/temps ouverts » (Fusulier \& Marqui, 2009: 6) et elles sont traversées par des rapports de pouvoir face auxquels les individus font des compromis (Rémy, 1996). 
L'approche des « sexualités négociées 》 (Combessie \& Mayer, 2013) permet d'approfondir le concept de négociation dans le contexte de la sexualité. À la suite de Zelizer (2001), qui avait déjà bien montré l'importance de ne pas dissocier les sphères économiques et intimes dans l'analyse des «transactions intimes", Combessie et Mayer (2013) proposent d'articuler dans l'analyse des transactions sexuelles, l'aspect financier, le monde des affects et le domaine de la reconnaissance. Tout en reprenant l'idée d'un continuum de Tabet (2004), entre transactions marchandes (activités de type prostitutionnel) et non-marchandes (comme le mariage), cette approche s'éloigne néanmoins d'une lecture en termes de rapports de domination pour privilégier une analyse en terme de négociations qui permet justement d'articuler ces trois registres en reconnaissant leur imbrication constante. Une telle analyse permet de reconnaître les enjeux de contrôle et de rapports de pouvoir mais aussi les marges de manœuvre.

Ainsi, nous refusons de réduire la sexualité à la domination masculine (Clair, 2013). Nos résultats montrent que le genre, compris comme une logique globale qui organise la société, et la sexualité sont articulés de façon étroite. En effet, les expériences des jeunes rencontré-e-s sont largement organisées en fonction de ce que Clair (2008) nomme « l'ordre du genre ». Pour cette chercheuse, cet ordre, qui repose sur un système hétéronormatif (Buttler, 2005), renvoie à la fois à la «mise en ordre (le classement) hiérarchique des groupes de sexe et le rappel à l'ordre des normes de masculinité et de féminité » (Clair, 2013 : 113) (nous y reviendrons). Dans ce système, en matière de sexualité, les hommes sont encouragés à faire preuve de leur désir, voire de leur besoin de sexualité (hétérosexuelle), alors que les femmes sont encouragées à faire preuve de retenue et de responsabilité. Tabet (2004) va plus loin et pose la sexualité des femmes comme étant davantage un service sexuel qu'une motivation propre. Nous retrouvons en effet dans les discours des jeunes la représentation sociale selon laquelle la sexualité des femmes doit (souvent) s'adapter à la demande masculine. Toutefois, nos résultats montrent que les femmes disposent de marges de 
manœuvre qui révèlent qu'elles ne sont pas non plus tout à fait « expropriées » de leur sexualité, comme le soutient Tabet. Ainsi, la perspective que nous proposons dans cet article est de considérer la sexualité féminine comme une "dette de sexe», dans le sens que, dans l'ordre du genre, le désir féminin apparaît subordonné au désir masculin. Cet ordre hétéronormatif constitue le cadre dans lequel les jeunes hommes et les jeunes femmes négocient, de façon plus ou moins contrainte, leurs engagements dans des transactions sexuelles.

Cet article porte sur les résultats d'entretiens individuels menés auprès de 37 jeunes ( 17 hommes, 18 femmes et 2 androgynes) de Suisse âgé-e-s de 14 à 27 ans $^{5}$. Ces entretiens semi-structurés ont abordé les représentations sociales et personnelles de la sexualité des jeunes, les relations amoureuses ou intimes et les expériences sexuelles, ainsi que les significations données aux transactions sexuelles vécues ou non. Cet article se concentre sur les expériences hétérosexuelles relatées par les jeunes, indépendamment de leur orientation sexuelle, dans lesquelles la logique de la redevabilité était la plus manifeste.

Afin de mettre en lumière le sens donné par les jeunes à leur expériences sexuelles, les données ont été analysées à l'aide d'une grille d'analyse (Karsz, 2004 ; Colombo et al., 2016) permettant d'opérationnaliser le concept de représentation à travers trois types de repères normatifs: les repères cognitifs (comment les jeunes comprennent-elles/ils les transactions sexuelles dans lesquelles elles/ils sont impliqué-e-s ?) ; les repères éthiques (en fonction de leurs valeurs que jugent-elles/ils acceptable ou non dans l'échange ?) et les repères politiques (comment se positionnent-elles/ils dans la négociation ?).

5 Pour plus d'informations concernant les caractéristiques de l'échantillon, voir Colombo, Carbajal et al., (2017). La récolte des données comprend également deux autres volets : un questionnaire en ligne ciblant des jeunes de 14 à 25 ans vivant en Suisse et des focus-groups auprès de professionnel-le-s. 


\section{Consentir à des expériences sexuelles par sentiment de redevabilité}

Lorsqu'on aborde la question des échanges associés à la sexualité, la plupart des jeunes rencontré-e-s pensent à la prostitution. Or, l'analyse des récits de leurs expériences montre que la logique "professionnelle», où la transaction renvoie à la rétribution de services sexuels dont les modalités sont négociées d'avance, est minoritaire. Les transactions sexuelles qu'elles et qu'ils relatent sont diversifiées et leur déroulement est rarement planifié d'avance. Ainsi, l'engagement des jeunes dans ces transactions correspond davantage à des compromis (Schurmans, 2013). Les jeunes qui s'y engagent disposent d'une marge de manœuvre plus ou moins importante et négocient les termes de l'échange, avec eux-mêmes et elles-mêmes, ainsi qu'avec l'autre/les autres, sur un continuum entre liberté et contrainte. Dans ces compromis, temporaires et susceptibles d'être remis en question, différentes logiques subjectives sont à l'œuvre et peuvent se combiner, comme des logiques de reconnaissance et des logiques de redevabilité.

Cet article s'intéresse plus particulièrement aux expériences où le compromis repose sur une logique de redevabilité. En effet, plusieurs jeunes, principalement des femmes, ont expliqué avoir accepté de s'engager dans des expériences sexuelles, avec ou sans lendemain, qui peuvent aller d'un baiser à des relations sexuelles avec pénétration, en passant par des caresses ou du sexe oral, sans forcément en avoir envie, mais parce qu'elles se sentaient redevables face à leur partenaire.

Une enquête récente menée en Suisse auprès de jeunes âgé-e-s de 26 ans en moyenne (Barense Dias, Akre et al., 2018) révèle que $53 \%$ des femmes interrogées ont accepté des relations sexuelles sans désir.

Plusieurs études auprès des jeunes (citées par Dubé et al., 2015) montrent que lors d'aventures sans lendemain, les jeunes femmes se retrouvent plus souvent que les jeunes hommes impliquées dans des expériences sexuelles qu'elles regrettent après coup, que plusieurs d'entre elles acceptent les avances des jeunes 
hommes pour éviter les conflits et qu'elles sont plus nombreuses que ces derniers à développer diverses stratégies pour réduire leur résistance ou leur anxiété face à des actes (comme des fellations par exemple) qu'elles consentent à réaliser surtout pour faire plaisir au partenaire. Riggenbach (2017) montre que même au sein de relations de couple plus stables, les femmes peuvent consentir à des relations sexuelles sans en avoir envie. Lorsque le désir sexuel diminue, certaines jeunes femmes se plient au désir du conjoint pour répondre à sa demande et faire exister le couple 6 .

L'extrait d'entretien avec Joan ${ }^{7}$ en introduction rend compte de ce sentiment d'obligation que peuvent ressentir les jeunes femmes, comme si, dans certaines circonstances, elles n'envisageaient même pas la possibilité de dire non à des propositions sexuelles de la part de jeunes hommes, même si elles n'en ont pas envie. Elle explique par exemple avoir accepté les avances d'un ami sans pourtant en avoir envie sur le moment. Elle passait beaucoup de temps chez lui, fuyant un domicile familial où elle ne se sentait pas à l'aise. Même si elle semble suggérer que cette expérience lui a aussi apporté des éléments positifs (« ce n’était pas mal du tout »), elle se rend compte après-coup qu'elle y a davantage consenti par sentiment de redevabilité :

Je me suis sentie redevable envers [lui]... [...] Parce que justement, je venais squatter chez lui hyper souvent. Et puis il m'a fait comprendre

${ }^{6}$ Parmi les expériences relatées par les jeunes rencontré-e-s dans le cadre de notre enquête, une minorité s'inscrivait dans des relations de couple décrites comme stables, même si parfois il s'agissait de relations avec un-e partenaire régulier et/ou connu-e. On peut faire l'hypothèse, à la suite de Zelizer (2001), que dans les représentations de plusieurs jeunes, les échanges renvoient à une logique marchande peu compatible avec la logique affective qui devrait (dans les représentations) prédominer dans une relation de couple stable. Or, l'intérêt de ces expériences est notamment qu'elles permettent d'analyser les raisons qui expliquent la logique de redevabilité indépendamment des enjeux liés à une relation de couple (se voulant) stable.

7 Joan se dit androgyne, mais nous reprenons le genre féminin qu'elle utilise pour parler d'elle et qui correspond au genre dans lequel elle a été socialisée. 
qu'il avait envie d'aller plus loin et tout ça. Et puis il y a eu un jour où j'ai séché les cours pour aller le voir et puis on s'est posés toute la journée chez lui, on a regardé des films, on a mangé des crêpes et tout. Et puis j'étais posée contre lui et puis au bout d'un moment, il a commencé à me caresser le bras et tout ça. Donc je sentais qu'il en avait envie et puis il a commencé à m'embrasser et puis sur le moment, j'en avais absolument pas envie. Et puis on est allés dans la chambre et puis finalement, c'était pas mal du tout. C'était pas le genre où je me suis sentie abusée, parce que c'est moi qui lui ai pris la main et puis on est allés dans la chambre. Mais sur le moment j'avais vraiment pas envie et j'étais là, mais pourquoi? (Joan, 17 ans).

On voit dans cet exemple que si Joan accepte la proposition sexuelle de son ami, ce n'est pas parce qu'elle en a envie, mais parce qu'elle estime qu'elle le lui doit, étant donné qu'il l'a hébergée. C'est comme si elle considérait sa sexualité comme un contre-don lui permettant de rétablir un certain équilibre dans la relation et de ne pas se sentir profiteuse. Le fait de rendre en offrant sa sexualité « est destiné à rétablir l'égalité compromise par le don » (Weber, 2000 : 96).

Ce que disent les jeunes filles rencontrées, c'est que si certaines d'entre elles acceptent de répondre aux attentes (exprimées ou présumées) des hommes qui leur offrent ce qu'elles considèrent comme une ou plusieurs faveurs, ce n'est pas tant parce qu'elles n'arrivent pas à dire non que parce qu'elles pensent qu'elles auraient dû savoir que les hommes qui offrent quelque chose à une femme ont toujours des attentes sexuelles. Par conséquent, elles auraient dû se douter qu'en acceptant ces faveurs, elles créeraient des attentes sexuelles chez eux. Estelle, 21 ans, raconte l'expérience d'une soirée, lorsqu'elle était plus jeune, où elle et deux amies ont rencontré trois jeunes hommes et elles ont été dormir chez l'un d'entre eux. Durant cette nuit, l'une des trois copines a eu une relation sexuelle pénétrative avec l'un des jeunes hommes :

Une fois, on était trois filles. On est allées à une soirée-mousse et puis on a rencontré trois gars. [..] Et puis du coup, les couples se sont formés direct. Une avec un, moi avec l'autre et encore une avec un autre. On est allés dormir les six chez un des gars. Ben il y en a une qui l'a fait avec un des gars. Ouais... enfin ça devait arriver. 
Vous étiez conscientes de ça en allant chez eux ?

Non, à ce moment-là, en tout cas personnellement j'étais pas consciente qu'il y avait possibilité de ça [...] Je pense que c'est vraiment un moment où... voilà, on va en boîte, on sait pas trop où on va dormir. Si c'est pas hyper planifié, c'est vrai qu'on est un petit peu plus susceptibles d'aller dormir chez quelqu'un parce que c'est pratique. Ouais, c'est pas genre : «Ah ce soir je vais en boîte et je vais dormir chez un étranger », enfin on n'y pense pas. C'est vraiment parce que ça tombe sur le moment, genre : «Mais moi j'ai de la place - Ah cool! ». On se jette dans la gueule du loup (Estelle, 21 ans).

En disant que «ça devait arriver », Estelle accepte avec un certain fatalisme le déplacement du sens donné à la proposition d'hébergement. D'une proposition spontanée visant à pallier l'absence de solution d'hébergement des filles et, peut-être, à la volonté de prolonger ensemble l'ambiance festive de la soirée, elle devient une proposition de transaction sexuelle une fois dans l'appartement. Cette proposition est négociée différemment par les jeunes femmes, faisant chacune le compromis lui semblant le plus acceptable ou attendu compte tenu du déroulement de la situation. Or, si Estelle n'avait pas conscience de cette possibilité en acceptant la proposition d'hébergement, elle ne semble pas envisager après coup que les choses aient pu se passer autrement. Comme l'ont montré Gagnon et Simon (1973), les comportements sexuels des individus dépendent d'apprentissages sociaux, transmis à travers la socialisation. Ces apprentissages sociaux participent à la construction de « scripts sexuels » qui font office de repères dans les interactions sexuelles. Or, le genre traverse les scripts sexuels en tant que « ... matrice de dispositions générales, comme cadre de socialisation différenciée à la sexualité, et comme guide instruisant» (Monteil, 2016). Dans ce sens, le fait d'accepter de se faire héberger par un homme s'inscrit dans un «script sexuel» où les hommes ont toujours des attentes sexuelles envers les femmes et les femmes qui acceptent une faveur ou des faveurs de leur part s'engagement implicitement à y répondre, qu'elles en aient envie ou non. C'est ce qu'exprime également Cléa. Même si elle ne se sent pas forcément 
obligée de répondre aux attentes des hommes qui lui paient des verres, elle ne remet pas en question le fait qu'un homme qui offre un verre à une femme attend qu'elle lui offre sa sexualité en retour :

On me paie souvent des verres, j'en profite, je suis étudiante [...]. Non, je veux dire si on m'offre un truc, on me demande rien en retour, ben tant mieux. Après est-ce que la personne attend quelque chose ? Oui sûrement. (Cléa, 18 ans).

Même si accepter un verre dans l'espace public comporte peut-être moins d'enjeu que d'aller dormir chez un homme, l'intention prêtée aux hommes est la même.

Lorsqu'ils relatent leurs expériences hétérosexuelles, aucun des jeunes hommes rencontrés n'exprime un tel sentiment de redevabilité. Non seulement ils ne relatent pas des situations où ils se sont sentis obligés d'offrir leur sexualité à une femme leur ayant donné des faveurs, mais certains d'entre eux ne semblent même pas se demander si celles-ci pourraient se sentir obligées de leur offrir leur sexualité en retour. Jonas, 17 ans, raconte une expérience où il a offert son toit à une fille qui ne savait pas où dormir suite à une soirée, sans attendre forcément ses faveurs sexuelles en retour. Ils ont finalement eu une aventure d'une nuit :

Il y avait une fête chez mon ami et la fille savait dès le début qu'il ne pouvait pas lui offrir une place pour dormir. Malgré cela, elle est venue et on a commencé à parler et elle m'avait demandé si j'avais de la place et si elle pouvait venir dormir chez moi et j'ai dit oui. On s'est bien entendu ce soir, on avait bu un peu et ensuite on est rentrés ensemble... et une chose a mené à l'autre. Mais pour moi ce n'est pas un échange, mais ça s'est développé en parallèle.

Ce n'était pas planifié dès le début?

Non. Alors ça, même de son côté à elle, ce n'était pas planifié que : « oui je peux dormir chez lui alors je dois coucher avec lui ». C'était pas avec une arrière-pensée. Après quand on était au lit tout à coup... oui [rire], tout à coup ça s'est fait ! (Jonas, 17 ans).

On voit que Jonas interprète l'engagement de la fille dans cette expérience comme un acte spontané où "une chose a mené à l'autre » et il semble écarter l'hypothèse qu'elle ait agit par redevabilité. 
D'autres jeunes hommes, par contre, évoquent l'hypothèse que dans certaines situations, leurs partenaires ont pu se sentir obligées de leur offrir leur sexualité par sentiment de redevabilité. Par exemple Julio, 22 ans, raconte l'expérience d'une soirée où il a rencontré une jeune femme qui lui avait demandé si elle pouvait dormir chez lui. Sur le moment, il a pensé que la fille lui exprimait son désir d'avoir une aventure sexuelle d'un soir avec lui. En y réfléchissant, il pense toutefois qu'elle s'est sentie obligée de s'engager dans une telle transaction, parce qu'il lui offrait un hébergement. Toutefois, malgré cette analyse, il ne semble pas remettre en question son engagement dans cette aventure :

Oui, j'étais en soirée. Je rencontre une personne avec qui je discute, je flirte et c'est cool, c'est agréable [...] Cette fille me demande si elle peut venir dormir chez moi. [...] Elle n'habitait pas [la ville où on était] et elle est venue avec moi chez moi. Je lui avais proposé que je dorme sur le sofa. Je lui ai dit qu'on n'est pas obligés de dormir dans le même lit. Elle a répondu que je pouvais sans autre dormir dans mon lit $[\ldots]$. Et après de cette situation s'est développé quelque chose entre nous. Et ensuite, je me suis dit qu'elle ne cherchait pas forcément à coucher avec moi [...]. Moi je voulais quelque chose d'elle, mais c'était plus le fait qu'elle cherchait à dormir chez moi [...]. Mais j'ai l'impression que pour elle, en tout cas au départ, elle cherchait plus à trouver un endroit où dormir et pas directement à coucher avec moi.

As-tu l'impression que la situation l'a obligée...?

Non pas obligée, mais ...

...mais amenée à te faire un plaisir ?

Oui, c'était ma première impression (Julio, 22 ans).

René, 25 ans, évoque une situation où il pense qu'une fille, qui venait chez lui pour fumer du cannabis, acceptait de lui faire des fellations non seulement par plaisir, mais surtout parce qu'il lui fournissait la drogue :

Quand on se voyait, on fumait ensemble et après il arrivait qu'on se faisait parfois encore des attouchements. Mais on n'avait pas du sexe, mais [...] des fellations et des trucs du style. Moi j'avais toujours quelque chose à fumer et d'abord on fumait ensemble et seulement après on se touchait. [...] Ça c'est pour moi une forme d'échange. En fait, ce n'est pas vraiment ça l'échange, mais on pourrait le voir 
comme ça. Je ne pense pas que cette fille serait restée avec moi seulement pour rester assise avec moi sur un banc. C'est parce que j'avais quelque chose à fumer. Je pense que c'était vraiment ça qui l'a attirée vers moi. Bon, après c'est aussi parce qu'elle pouvait encore faire des choses excitantes avec moi. Mais je pense qu'il y a plusieurs aspects qui entrent en jeu. Le fait seulement d'être excitée par une personne, ça ne suffit pas à mon avis. Il faut que tu puisses lui donner encore d'autres choses (René, 25 ans).

On trouve dans les propos de René l'idée de Tabet (2004) selon laquelle la sexualité des femmes serait dépossédée de motivation propre, ce qui expliquerait que les hommes doivent acheter cette motivation en leur offrant des faveurs (comme la drogue dans ce cas) en contrepartie de leur sexualité. Or, cet exemple permet également de nuancer cette analyse : si René pense que la motivation principale de la fille était la drogue, il n'exclut pas l'excitation sexuelle.

Ces exemples montrent que si les jeunes femmes se sentent obligées de répondre aux attentes sexuelles présumées des jeunes hommes, ces derniers sont eux aussi soumis à des attentes de comportement sexuel. C'est comme si eux non plus ne remettaient pas en question le faible appétit sexuel des femmes («ça ne suffit pas »), d'une part et d'autre part, le fait que s'ils offrent un verre, un toit, voire de la drogue à une femme, cela signifie forcément qu'ils ont des attentes sexuelles envers elle. Ainsi, les jeunes hommes semblent accepter leur propre «dette de sexe», celle d'avoir constamment des envies sexuelles et d'être toujours disponibles, volontaires et performants sexuellement.

Ces exemples montrent que pour négocier leurs engagements dans des transactions sexuelles, les jeunes se réfèrent à des « scripts sexuels » qui correspondent à des attentes de comportements qui sont très différentes selon le genre. Dans ce sens, la logique de la redevabilité exprimée par les jeunes peut être comprise comme révélatrice de rapports sociaux de genre qui les dépassent. 


\section{La logique de redevabilité, révélatrice de l'asymétrie des rapports sociaux de genre}

La sexualité, comme d'autres pratiques sociales, peut être comprise comme un espace où les rapports de sexe se matérialisent (Clair, 2013). Ainsi, si les jeunes femmes interrogées se sentent davantage redevables de sexe que les jeunes hommes, c'est qu'elles et ils sont soumis à des attentes de comportements qui s'inscrivent dans un contexte social où prédomine un système de représentations binaires de la sexualité que Butler (2005) nomme « l'hétéronormativité ». Ce système normatif établit une correspondance linéaire entre sexe, genre et hétérosexualité. Dans cette logique où l'hétérosexualité est la norme, les rôles des hommes et des femmes au niveau de la sexualité sont compris comme étant différents et complémentaires.

À cette logique correspond des processus de socialisation différenciés selon le genre (Dafflon Novelle, 2006). Alors que les hommes apprennent à être forts, rationnels, volontaires, courageux, les femmes apprennent à être fragiles, douces, dévouées, crédules, pudiques, (Héritier, Perrot, Agacinscki \& Bacharan, 2011). Au niveau de la sexualité, cela se traduit par la représentation d'une sexualité masculine caractérisé par l'assertivité, la performance sexuelle, la virilité et le désir sexuel associé aux besoins physiologiques. La sexualité féminine devrait quant à elle être d'ordre relationnel, liée à l'affectivité et à la conjugalité (Bajos, Ferrand, \& Andro, 2008 ; Déroff, 2007). Dans ce système de représentations, la sexualité féminine est pensée comme une "dette de sexe», en réponse aux besoins impérieux des hommes. Selon une enquête française (Bajos, Ferrand, \& Andro, 2008), 73\% des femmes et 59\% des hommes français adhèrent à la croyance selon laquelle "par nature, les hommes ont plus de besoins sexuels que les femmes ». Toujours selon cette enquête, cette croyance a des incidences sur les pratiques sexuelles des femmes qui reconnaissent accepter davantage d'avoir des rapports sexuels sans en avoir envie. C'est également ce qu'exprime par exemple Joan lorsqu'elle dit avoir 
consenti à une relation sexuelle avec son ami parce que «c'est tout ce qu'il attendait ».

Ces croyances peuvent affecter d'autant plus les jeunes femmes dans le contexte d'une société qui encourage les jeunes à l'expérimentation sexuelle (Bozon, 2012). En effet, si les attentes de comportements face aux jeunes hommes sont compatibles avec la logique d'une sexualité libérée, celles adressées aux jeunes femmes les enferment dans une injonction paradoxale, puisqu'on attend d'elles à la fois qu'elles expérimentent librement, mais qu'elles soient responsables et inscrivent leur sexualité dans la conjugalité (Colombo, Carbajal et al., 2017). Comme l'explique Estelle, même si elle n'a pas forcément envie d'une relation sexuelle (l'utilisation du terme «niquer» renvoie même à une relation dégradante), elle peut être tentée d'accepter des propositions d'hébergement d'un homme pour ne pas passer pour une fille coincée :

Dire non [à un mec qui te propose de t'héberger après une sortie en boîte], ben c'est vrai que quand on dit non, ben on est le con de l'histoire. On est : "Ah, elle a pas voulu parce que... ». Quand on dit non à quelque chose, c'est qu'on est fermé, c'est qu'on est con et puis voilà. Dire oui, ça fait bien. Mais après, faut assumer ce qui se passe. Et après, c'est vrai qu'aller chez quelqu'un en se disant : « je suis allée chez lui, mais je sais très bien que je vais me faire niquer », c'est affreux. C'est affreux. Et puis le dire comme si on l'admet, enfin... on le sait, on le sait, mais on y va quand même (Estelle, 21 ans)

Or, ce que montrent ces exemples, c'est que non seulement les jeunes ne sont pas aussi libres que cela dans la négociation de leurs compromis, mais que par leurs comportements, elles et ils contribuent à reproduire cet ordre hétérosexuel. Femmes et hommes se rejoignent dans la complémentarité de leurs «dettes de sexe », mais dans un rapport hiérarchisé : les femmes pensent ne pas avoir d'autre choix que d'offrir leur sexualité en réponse aux attentes présumées des hommes, auxquels elles confirment qu'ils n'ont pas d'autre choix que de se montrer désirants, disponibles sexuellement et performants. De ce fait, elles et ils reproduisent $l^{\prime}$ « ordre du genre » (Clair, 2008). 
Toutefois, comme l'exprime Joan, les jeunes femmes ne consentent en fait pas «à $100 \%$ ». À la différence d'une transaction monétaire, instantanée, qui serait scellée par l'argent, dans le cas du don, la relation est ouverte : «C'est la dette qui fait que la relation ne s'arrête pas là, qu'elle n'est pas ponctuelle, fermée sur ellemême, complète [...]. La relation est ouverte, et dans l'ouverture s'engouffre la dette. »(Godbout, 2006 : 93). Ainsi, l'implicite peut renforcer le sentiment d'obligation, mais il peut aussi laisser place à une indétermination sur la nature du rendu, qui laisse dès lors la liberté de le négocier.

\section{Marges de manœuvre des jeunes et stratégies de négociation}

Le cas de Joan montre que sa sexualité constitue non seulement une dette, mais également une ressource qui lui permet d'alimenter le circuit de dons et contre-dons dont elle tire des avantages d'ordre matériel (hébergement, nourriture, etc.) et symbolique (sentiment de reconnaissance, protection). Elle n'est donc pas dans un rapport unilatéral, mais bien réciproque et elle conserve une marge de liberté et d'initiative, malgré le sentiment de redevabilité. Ces «transferts doubles» (don, contre-dons), non simultanés, se composent d'un enchaînement d'interactions (Weber, 2000 : 87) qui donnent lieu à un système de dépendances constitutives des relations sociales (Carré \& Loute, 2016), dans lequel les actrices et acteurs conservent une certaine liberté pour négocier la suite de la transaction. Deschamps (2013) montre par exemple que dans le cas de verres payés par des hommes aux femmes dans des bars populaires, ce sont la plupart du temps les femmes qui prennent l'initiative de la réponse et qu'en acceptant ces verres, elles cherchent à jouer à l'objet, éprouvant une sorte de plaisir à se faire désirer.

Dans plusieurs expériences relatées par les jeunes interviewée-s, les femmes prennent elles aussi l'initiative de la réponse, offrant parfois leur sexualité, mais aussi des moments de discussion ou un baiser. Comme on l'a vu plus haut, Cléa explique être consciente des attentes implicites des jeunes hommes qui lui paient des verres ou lui 
font des invitations. Lorsque qu'elle accepte des invitations ou des verres, elle refuse de donner sa sexualité, mais elle offre parfois des moments à passer ensemble et de la discussion :

On me paie souvent des verres, j'en profite, je suis étudiante [...]. J'ai un ami qui est comme ça, qui essaie toujours, qui m'offre des verres, qui m'invite à des soirées [...] je lui ai toujours dit clairement que «De mon côté, non, tu ne m'attires pas, je suis désolée, il se passera jamais rien entre nous », je lui ai dit et redit et redit. Et s'il continue à m'offrir des verres et puis à espérer quelque chose, c'est son problème [...] S'il me demande rien clairement, moi je pars du principe que c'est non. Enfin c'est... J'ai peut-être un petit côté un peu... opportuniste ! (Cléa, 18 ans).

Dans cet exemple, Cléa joue sur la clarté afin de bien signifier au créancier que l'acceptation du verre ne correspond pas à une disponibilité sexuelle. Ainsi, la clarté de sa position est, pour elle, une manière de se libérer de la responsabilité d'autrui, surtout si sa position à lui n'est pas aussi claire. En disant qu'elle « profite » et en se qualifiant d' " opportuniste», on peut penser qu'elle exprime le fait qu'elle se rend bien compte que, ce faisant, elle s'écarte des attentes de comportements adressés aux femmes et peut apparaître comme une profiteuse. Mais on peut aussi interpréter ces propos comme le fait qu'elle tire également des avantages de ce type de transaction, qui peuvent être d'ordre monétaire (se faire payer des verres), mais aussi d'ordre symbolique, comme par exemple, le fait d'obtenir de la reconnaissance en se sentant objet de désir sexuel.

Dans un autre exemple relaté par cette jeune femme, elle affirme encore davantage sa marge de manœuvre en disant s'être laissée embrasser en échange d'un verre par choix, parce que le jeune homme lui plaisait. $\mathrm{Si}$, dans un premier temps, elle ne semble pas avoir la possibilité d'éviter ce baiser, c'est bien elle qui a pris l'initiative de mettre fin à la relation immédiatement après, même si elle est consciente que le fait de se laisser embrasser aurait pu être compris comme un consentement à la transaction proposée :

J'ai jamais dit non à un verre, ça c'est vrai. Il me paie un verre, machin. Club bondé, il se penche pour m'embrasser et pas moyen de... machin. Après, ben il était mignon, c'était pas dérangeant. J'ai pas pris ça comme un verre contre un bisou, même si je pense que 
c'est plus ou moins l'idée. Je m'en rends bien compte. Mais moi ça m'a pas dérangée qu'il m'embrasse. [..] La seule chose que je lui ai dit après ça, c'était : « Tu sais où sont les chiottes ? » et ça s'est arrêté là. Il m'a rien demandé de plus (Cléa, 18 ans).

L'exemple de Cléa montre que les jeunes femmes ne font pas que reproduire l'ordre hétérosexuel et qu'elles peuvent aussi développer des réponses subversives face aux attentes de rôles genrés. En se montrant assertive à la fois sur le plan verbal et dans son attitude, Cléa, loin de se soumettre à l'obligation de contre-don, s'approprie un comportement socialement associé au genre masculin dans la logique de l'ordre hétérosexuel. En laissant peu de place à l'implicite et aux malentendus, Cléa court-circuite le sentiment de redevabilité. De cette manière, elle adopte les mêmes conditions que les jeunes hommes pour négocier avec eux. L'adoption des comportements associés au genre masculin est une manière, pour Cléa, de répondre à l'injonction faite aux femmes d'exprimer clairement leurs limites. Cet exemple montre toutefois que cette injonction ne peut avoir qu'un effet limité à des cas individuels, car elle demande aux jeunes femmes de changer leur comportement individuel sans agir sur l'ordre hétérosexuel au sein duquel prennent place ces comportements.

\section{Conclusion}

À travers l'analyse de quelques expériences de transactions sexuelles relatées par les jeunes rencontré-e-s dans le cadre de notre enquête, nous avons cherché à montrer que leurs engagements sont à comprendre comme des processus interactifs (non-unilatéraux) et dynamiques dans lesquels elles et ils négocient des compromis. L'apparente « libéralisation des mœurs » semble encourager aussi bien les jeunes femmes que les jeunes hommes à expérimenter davantage, notamment dans le domaine de la sexualité, et à prendre des initiatives dans le cadre de scripts sexuels qui se veulent plus égalitaires (Bozon, 2012). Or, nos résultats soulignent, comme d'autres travaux (voir notamment Clair, 2008), que la sexualité demeure un lieu où se manifestent de façon exacerbée les inégalités 
de genre. Dans cet article, nous avons particulièrement mis en lumière le fait que plusieurs jeunes femmes acceptent de s'engager dans des transactions sexuelles par sentiment de redevabilité face aux hommes. Or, nous avons montré que cette logique de redevabilité est moins à comprendre en termes de responsabilité individuelle que comme révélatrice de logiques associées à un « ordre du genre », fondé sur l'hétéronormativité, qui assigne des places sociales différentes aux hommes et aux femmes et qui se manifestent notamment à travers des injonctions de comportements sexuels.

Ces résultats nuancent la tendance actuelle qui renvoie le consentement sexuel à la seule responsabilité individuelle d'affirmer ses droits, notamment celle des femmes (Burkett \& Hamilton, 2012). En effet, nos résultats invitent plutôt à comprendre le consentement comme un compromis, temporaire et susceptible d'être remis en question, qui est négocié dans le cadre d'un « ordre du genre ». Cet ordre normatif engendre des « dettes de sexes » aussi bien féminines et masculines. Si les jeunes femmes se retrouvent plus souvent que les jeunes hommes à accepter des transactions sexuelles nonsouhaitées, ce n'est pas tant parce qu'elles savent moins dire «non » qu'eux, que parce que dans «l'ordre du genre», la sexualité féminine est posée comme une «dette de sexe» qui amène les jeunes femmes à se sentir redevables face aux attentes sexuelles des hommes. Or, en consentant à des transactions sexuelles sans forcément le désirer, elles confirment aux hommes leur propre "dette de sexe», qui est celle d'assurer une sexualité assertive, déterminée et désirante, et qui les amène parfois à faire preuve d'un (apparent) détachement face aux demandes des femmes.

Mais nous avons montré que ces jeunes ne font pas que reproduire l'ordre du genre de manière mécanique. Même si les jeunes femmes s'engagent parfois dans des transactions sexuelles sans le désirer, elles peuvent aussi y trouver des avantages voire, parfois, développer des réponses subversives aux attentes de rôles imposées par l'ordre du genre. Ainsi, le consentement est à comprendre comme un processus de négociation caractérisé par la 
tension entre la conformité aux normes de genre et la capacité de négocier des individus (Powell, 2008).

Il n'en reste pas moins que l'injonction actuelle faite aux jeunes d'affirmer de façon plus responsable leurs droits sexuels (IPPF, 2008) s'avère davantage cohérente avec les attentes sociales de comportement sexuel adressées aux hommes que celles adressées aux femmes. En attendant des femmes qu'elles affirment individuellement leurs droits sexuels, dont le droit au consentement, sans agir sur les conditions de réalisation de ces droits ni sur les processus genrés qui les déterminent, on court le risque de renforcer leur sentiment de redevabilité plutôt que de les aider à s'en émanciper.

\section{RÉFÉRENCES BIBLIOGRAPHIQUES}

BAJOS N., FERRAND M. \& ANDRO A., 2008. «La sexualité à l'épreuve de l'égalité », in BAJOS N. \& BOZON M. (dir.), Enquête sur la sexualité en France. Pratiques, genre et santé. Paris, La Découverte : 545-576.

BOZON M., 2002. Sociologie de la sexualité. Paris, Nathan.

BOZON M., 2012. "Autonomie sexuelle des jeunesse et panique morale des adultes. Le garçon sans frein et la fille responsable », Agora débats/jeunesse, 60(1) : 121-134.

BROQUA Ch., DESCHAMPS C. (dir.). 2014. L'échange économico-sexuel. Paris, EHESS.

BURKETT M., HAMILTON K., 2012. "Postfeminist Sexual Agency: Young Women's Negotiations of Sexual Consent", Sexualities, 15(7): 815-833.

BUTLER J., 2005 [1990]. Troubles dans le genre. Pour un féminisme de la subversion. Paris, La Découverte.

COMBESSIE Ph., MAYER S., 2013. «Une nouvelle économie des relations sexuelles? », Ethnologie française, 43(3) : 381-389. 
CARRE L., LOUTE A., 2016. "Introduction. Don et reconnaissance en débats », in CARRE L., LOUTE A. (dir.), Donner, reconnaitre, dominer. Trois modèles en philosophie sociale. Villeneuve d'Ascq, Presses universitaires Septentrion : 9-23.

COLOMBO A., REYNAUD C. \& DE COULON G., 2016. "Begging in Geneva: Which Right to the City?", Environnement Urbain/Urban Environment

[Online], 10, http://eue.revues.org/1306.

COLOMBO A., CARBAJAL M., CARVALHO BARBOSA M. \& TADORIAN M., 2017. "Gagner la reconnaissance des pairs en évitant la réputation de "pute". L'injonction paradoxale qui pèse sur les filles impliquées dans des transactions sexuelles », Revue Jeunes et Société, 2(2) : 70-93.

http://rjs.inrs.ca/index.php/rjs/article/view/117/69.

CLAIR I., 2008. Les jeunes et l'amour dans les cités. Paris, Armand Colin.

CLAIR I., 2013. « Pourquoi penser la sexualité pour penser le genre en sociologie ? Retour sur quarante ans de réticences », Cahiers $d u$ Genre54(1) : 93-120.

DAFFLON NOVELLE A., 2006 (dir), Filles-garçons : socialisation différenciée? Grenoble, PUG.

DEROFF M.-L., 2007. Homme/Femmes : la part de la sexualité. Une sociologie du genre et de l'hétérosexualité. Rennes, PUR.

DESCHAMPS C., 2013. "Prix et valeur dans la circulation du désir », Ethnologie française, 43(3) : 391-398.

DUBE S., LAVOIE F., BLAIS M. \& HEBERT M., 2015. «L'aventure sans lendemain chez les adolescents hétérosexuels: réflexions et pistes d'intervention», Revue québécoise de psychologie, 36(1) : 105-126.

FÉDERATION INTERNATIONALE POUR LA PLANIFICATION FAMILIALE (IPPF), 2008. Déclaration des droits sexuels de l'IPPF. Londres. 
FUSULIER B., MARQUI N., 2009. «Faire une sociologie de la transaction sociale ou de la transaction sociale une sociologie ? Quelques précisions pour répondre à l'interpellation de Maurice Blanc », Recherches sociologiques et anthropologiques, 2 : 141-147.

GAGNON J., SIMON W., 1973. Sexual Conduct: The Social Sources of Human Sexuality. NewBrunswick (USA)/London (UK), AldineTransaction.

GODBOUT J., 2006. "Le don au-delà de la dette», Revue du MAUSS, 27(1) : 91-104. DOI 10.3917/rdm.027.0091

HÉRITIER F., PERROT M., AGACINSKI S. \& BACHARAN N., 2011. La Plus Belle Histoire des femmes. Paris, Seuil.

JODELET D., 1989. "Représentations sociales: Un domaine en expansion, in JODELET D. (dir.), Les représentations sociales : 4778. Paris, PUF.

KARSZ S., 2004. Pourquoi le travail social ? Définition, figures, clinique. Paris, Dunod.

MONTEIL L., 2016. " Scripts sexuels », in RENNES J. (dir.), Encyclopédie critique du genre. Paris, La Découverte : 584-595.

POWELL A., 2008. "Amor fati? Gender habitus and young people's negotiation of (hetero)sexual consent", Journal of Sociology, 44(2): 167-184.

REMY J., 1996. «La transaction, une méthode d'analyse: contribution à l'émergence d'un nouveau paradigme $»$, Environnement \& Société, $17: 9-31$.

RIGGENBACH M., 2017. Le couple, la sexualité et l'amour. L'expérience des relations sexuelles consentantes sans désir des femmes à l'aube de leur vie amoureuse. Master en sciences sociales, pilier sociologie. Travail de Mémoire. Université de Neuchâtel.

SCHURMANS M.-N., 2013. "Négociations et transactions: un fondement socio-anthropologique partagé », Négociations, 20(2) : 81-93.

TABET P., 2004. La grande arnaque. Sexualité des femmes et échange économico-sexuel. Paris, L'Hamattan. 
WEBER F., 2000. "Transactions marchandes, échanges rituels, relations personnelles. Une ethnographie économique après le Grand Partage », Genèses, 41(4) : 85-107. DOI 10.3917/gen.041.0085

ZELIZER V., 2001. "Transactions intimes », Genèses, 42(1): 121-144.

\section{Résumé}

Cet article analyse la logique de redevabilité exprimée par plusieurs jeunes femmes impliquées dans des expériences de transactions sexuelles. Cette logique est révélatrice d'attentes sociales associées à un ordre hétéronormatif, qui influencent les comportements sexuels. En prenant appui sur une recherche menée auprès de jeunes âgé-e-s de 14 à 25 ans en Suisse, le consentement sexuel ne se réduit pas à la capacité individuelle à dire « oui » ou «non». Il est à comprendre comme un "compromis» réalisé par les jeunes, temporaire et susceptible d'être remis en question. Ce compromis est négocié dans le cadre d'un " ordre du genre » où la sexualité féminine est posée comme une « dette de sexe».

Mots-clefs : transactions sexuelles, redevabilité, jeunes, consentement, hétéronormativité, dettes de sexe.

\section{Summary}

Consenting to Unwanted Sexual Experiences

The Logic of Indebtedness: Individual Responsibility or Gendered Social Expectations?

This paper analyzes the logic of indebtedness as expressed by several young women involved in sexual experiences. This logic reveals social expectations related to a heteronormative order that influences sexual behavior. Research on sexual transactions involving young people aged 14 to 25 in Switzerland shows that sexual consent is not simply the individual ability to say "yes" or "no". It is to be understood as a temporary "compromise" made by young people, one that can be revisited. This compromise is negotiated in the context of a "gender order" where female sexuality appears as a "sexual debt".

Key-words: sexual transactions, indebtedness, youth, consent, heteronormativity, sexual debt. 